EPJ Web of Conferences 92,02077 (2015)

DOI: $10.1051 /$ epjconf/ 20159202077

(C) Owned by the authors, published by EDP Sciences, 2015

\title{
Numerical simulation of pump-intake vortices
}

\author{
Pavel Rudolf ${ }^{1, a}$, Roman Klas ${ }^{1}$ \\ ${ }^{1}$ Brno University of Technology, Faculty of Mechanical Engineering, V. Kaplan Department of Fluid Engineering, \\ Technicka 2896/2, Brno, Czech Republic
}

\begin{abstract}
Pump pre-swirl or uneven flow distribution in front of the pump can induce pump-intake vortices. These phenomena result in blockage of the impeller suction space, deterioration of efficiency, drop of head curve and earlier onset of cavitation. Real problematic case, where head curve drop was documented, is simulated using commercial CFD software. Computational simulation was carried out for three flow rates, which correspond to three operating regimes of the vertical pump. The domain consists of the pump sump, pump itself excluding the impeller and the delivery pipe. One-phase approach is applied, because the vortex cores were not filled with air during observation of the real pump operation. Numerical simulation identified two surface vortices and one bottom vortex. Their position and strength depend on the pump flow rate. Paper presents detail analysis of the flow field on the pump intake, discusses influence of the vortices on pump operation and suggests possible actions that should be taken to suppress the intake vortices.
\end{abstract}

\section{Introduction}

Proper operation of the centrifugal pump does not only depend on its design, but also on correct location within the sump. It is well known that placing pump suction throat too close to bottom or to side walls can induce non-uniform flow with strong vortices [1]. These phenomena have detrimental effect on pump properties due to increase of unsteady effects (e.g. flow pulsations), flow blockage, inception of cavitation in vortex core or at impeller leading edge. Lots of experimental research has been carried out on scaled models of pumps and intakes [2-10]. Computational simulations were prohibited for quite a long time because requirements on computational mesh resolution and turbulence modelling were relatively high. While today CFD modelling of pump intakes become feasible it is still a challenge and body of literature is available, where different computational approaches are tested with different success [11-16].

\section{Case description}

Chemical company in the Czech Republic encountered problems with drop of pump head and efficiency curves even around the best efficiency point (BEP). Pump was operated with sufficient suction head. Later tests in the pump manufacturer's laboratory pointed to improper placement of the pump within the sump as possible cause of the operation deterioration. However no typical intake, air filled vortices were observed.
Vertical centrifugal radial one-stage pump with guide vanes and annular diffuser is used for transport of clean cold water from main sump to different operating units. Main sump is divided into 3 parts, which are connected by rectangular holes $(2000 \times 1000 \mathrm{~mm})$, see figure 1 . Inlet pipe to the first sump has diameter $1000 \mathrm{~mm}$. The sump is not completely filled, water creates open level with approximately constant depth $(2600 \mathrm{~mm})$ above sump bottom. Suction throat of the pump is placed $1130 \mathrm{~mm}$ above the bottom. Model in figure 1 was used to obtain inlet velocity profile to the last part of the sump. Then, only this last part was modeled (from now on referred as reduced model), see figure 2 .

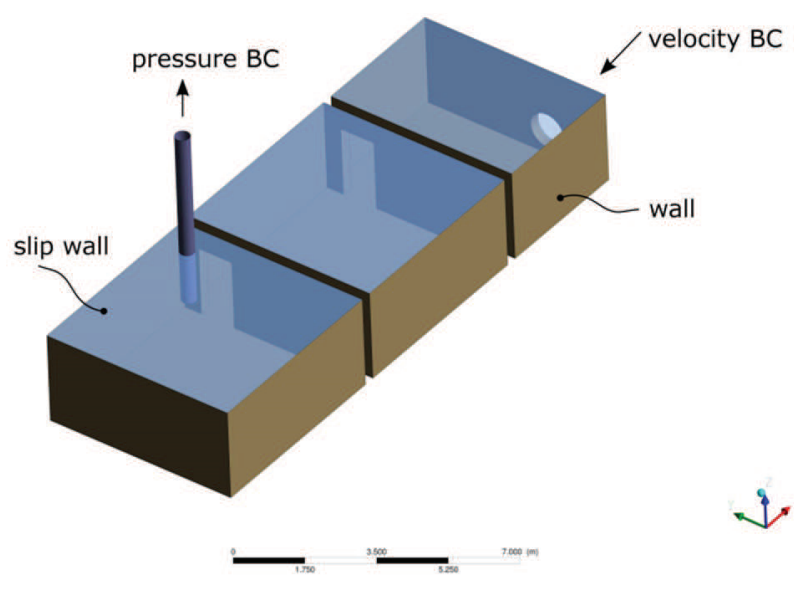

Figure 1. Series of sumps, pump is submerged in the last sump.

\footnotetext{
${ }^{\mathrm{a}}$ Corresponding author: rudolf@fme.vutbr.cz
} 
Reason for the model reduction was to have sufficiently fine mesh around the pump to capture all vortical structures, which would not be possible on the full size model due to prohibitive computational expanses.

Reduced model comprises the last part of pump sump together with stator case of the pump, inlet suction cone and inlet supporting cross (partial suppression of preswirl, figure 3) and outlet pipe. Pump impeller is not included. Since the vortical structures appear even around BEP, they cannot be primarily caused by impeller induced swirl.

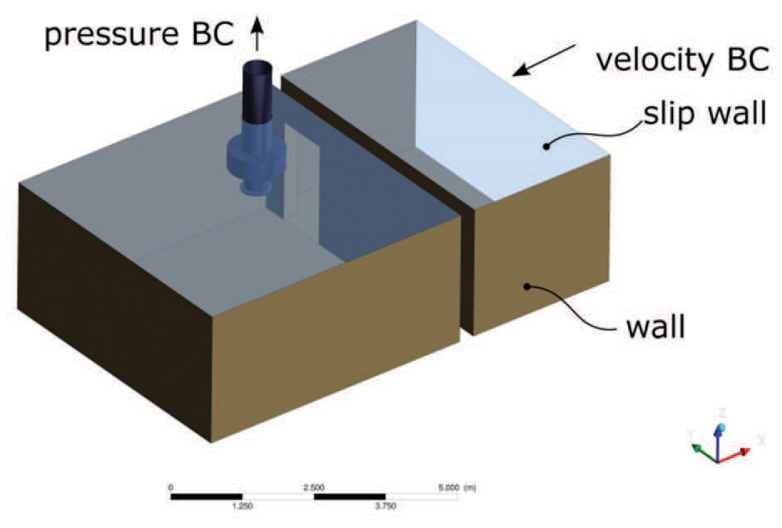

Figure 2. Reduced computational model with only the last sump with vertical pump.

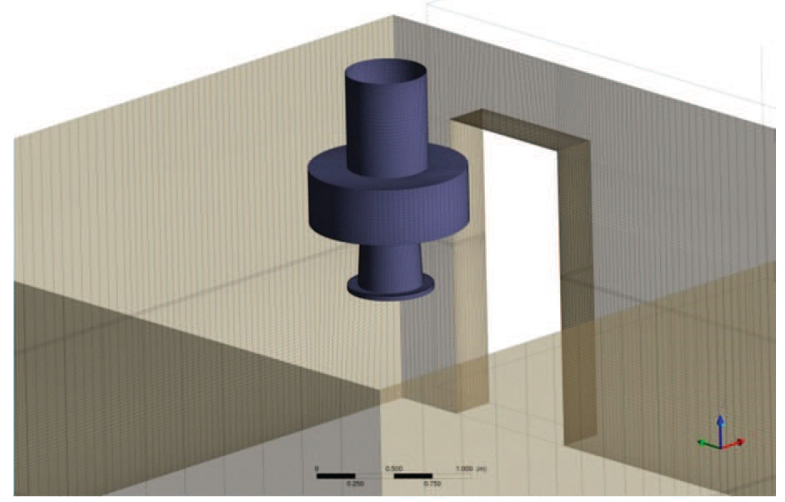

Figure 3. Detail of the pump and its position within sump, surface mesh is illustrated.

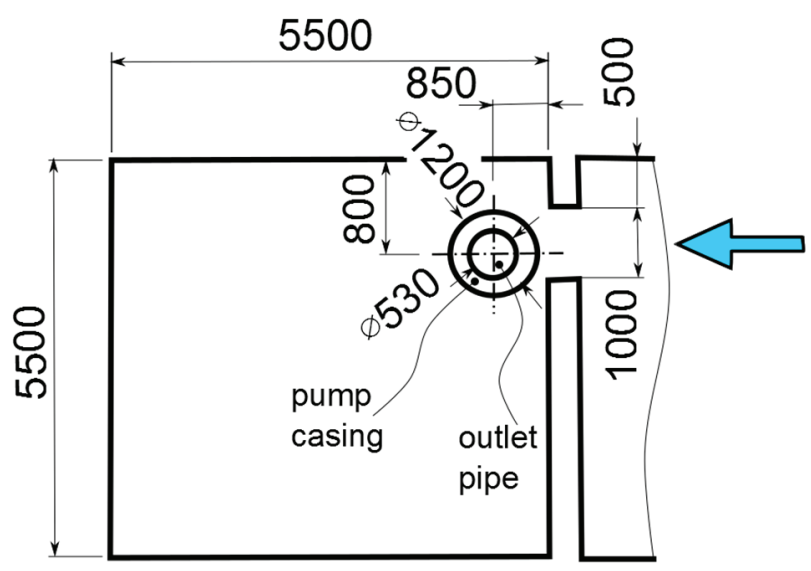

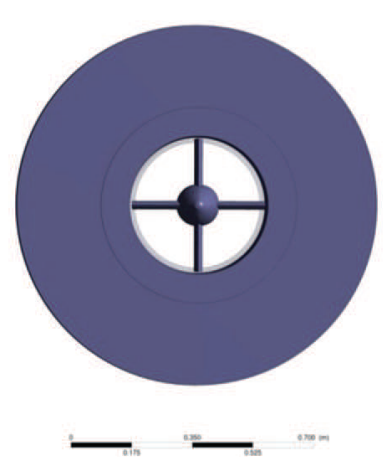

Figure 5. View of the pump suction with supporting cross.

Typical three operating points $(55 \%$ of BEP flow rate, BEP flow rate, $115 \%$ of BEP flow rate, $Q_{B E P}=555 \mathrm{l} / \mathrm{s}$ ) were simulated to obtain information about behavior of the vortices with respect to flow rate.

\section{Computational modeling}

Computational mesh of the spatially reduced model (figure 3) was divided using block scheme and filled with hexahedral mesh to ensure better accuracy. Mesh was clustered around and inside the suction cone, where appearance of the vortices was assumed. Whole mesh has 4.3 million cells.

Velocity boundary condition on the inlet to the domain and turbulence quantities were transposed from the full model (figure 1) with much coarser mesh. Constant average pressure was prescribed on the outlet of the delivery pipe. All sump walls and pump casing surface were modelled as no-slip walls, whereas water level is simulated by slip wall. It is proved by many authors that omitting phase interface at water level does not prevent capturing of the vortices, while severely reducing computational complexity.

Turbulence modelling presents an important issue when dealing with swirling flows, streamline curvature and need for resolving the coherent structures. It was shown in previous papers of the present author [17] that Reynolds Stress Model (RSM) is a suitable choice for this class of flows. It is argued by Skerlavaj et al [11] that while RSM performs well in presence of turbulence anisotropy it is rather computationally expensive compared with variants of SST model modified by different curvature correction formulas. It is especially recommended to use SAS-SST model, while large eddy simulation (LES) approach is claimed to be enormously costly and poses severe requirements on mesh quality. Therefore Scale Adaptive Simulation (SAS) model within ANSYS Fluent 14.5 was adopted in present paper for modeling of the vortices at pump suction. SAS provides better resolution of unstable transient turbulent structures (e.g. flow separations) without explicit mesh or time-step dependency.

Figure 4. Top view of the pump sump with dimensions. 


\section{Results}

Simulation results were studied qualitatively and quantitatively. CFD modelling revealed that for all three operating points three vortices are present, which extend to the suction space of the pump. Namely, there are two surface vortices and one bottom vortex. Figures 6-8 depict streamlines released either from water surface or from surface close to bottom.

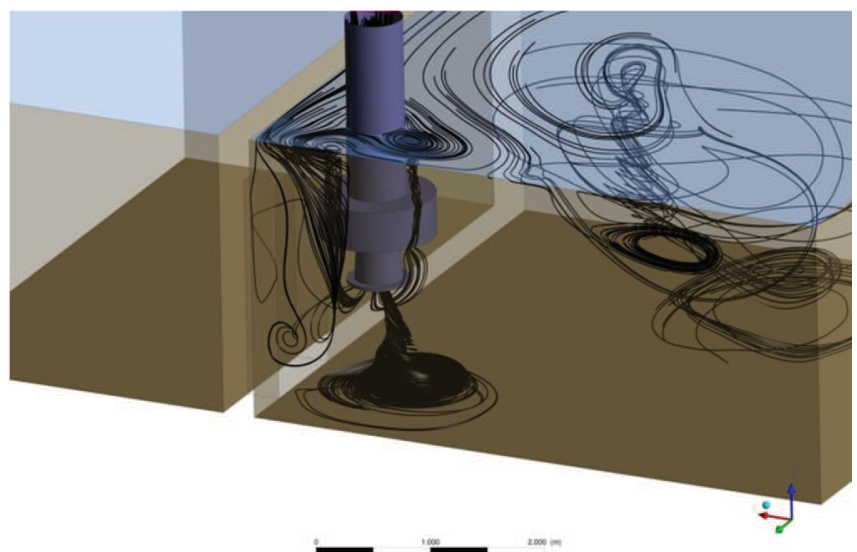

Figure 6. Streamlines visualizing vertical structures for $\mathrm{Q}=55 \%$ of BEP flow rate.

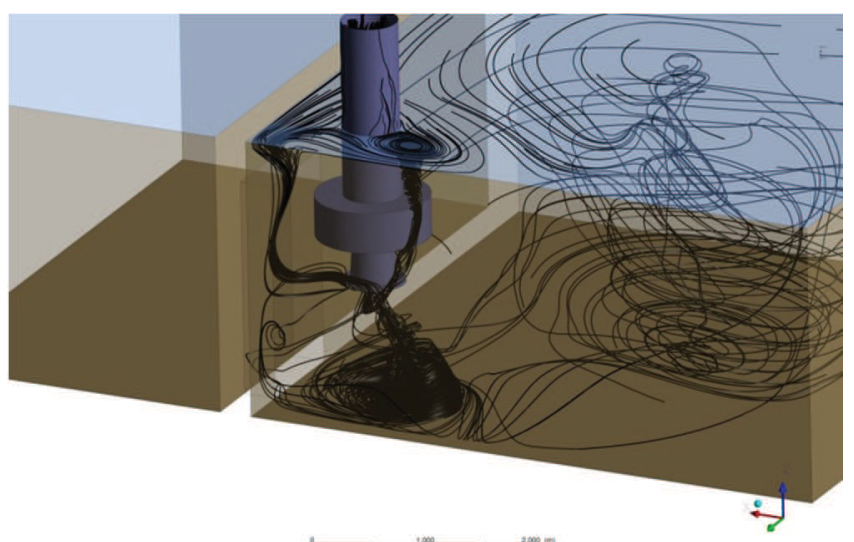

Figure 7. Streamlines visualizing vertical structures for $\mathrm{Q}=100 \%$ of BEP flow rate.

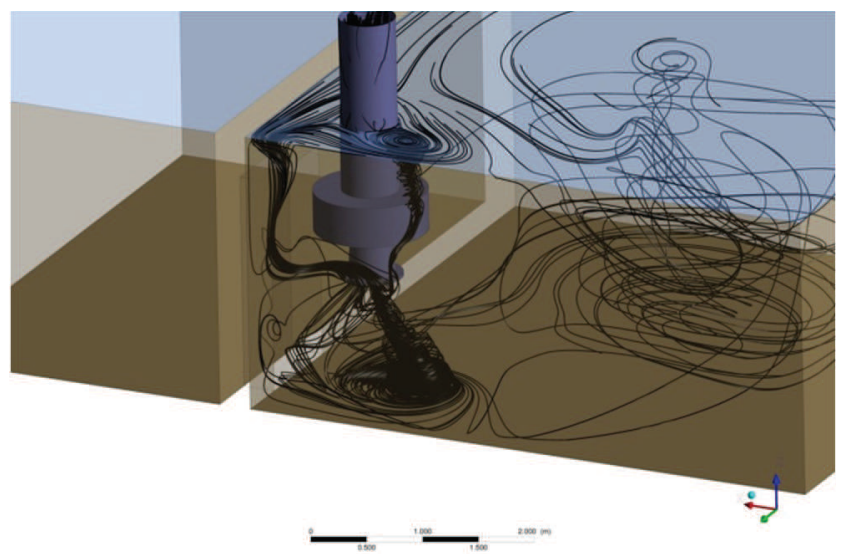

It is apparent that the streamlines are rolled into spirals and then sucked into pump. Streamlines released from surfaces close to sides of the sump did not show such behavior. Actual position and strength of the vortices depends on the operating point, i.e. flow rate, as will be further discussed.

Flow into the pump impeller is divided into four parts (quadrants) by the supporting cross. While initial idea pointed to partial blockage of one of the quadrants by the vortices, it was later proved that difference in mass flux among the quadrants is up $2 \%$, i.e. negligible.

Further analysis focused on non-uniformities of axial and circumferential velocity components. Only results for the best efficiency point (BEP) flow rate are presented. Two cross-sections were selected: inlet to the suction cone and position at the end of the supporting cross, i.e. just in front of the impeller. Figures show that both positive and negative circumferential velocities are present, which is a sign that vortices occupy part of the cross-section. It is interesting that axial velocity field is more deformed at impeller inlet than at the inlet to suction cone. This fact is most probably due to vortex stretching and strong velocity components coupling.

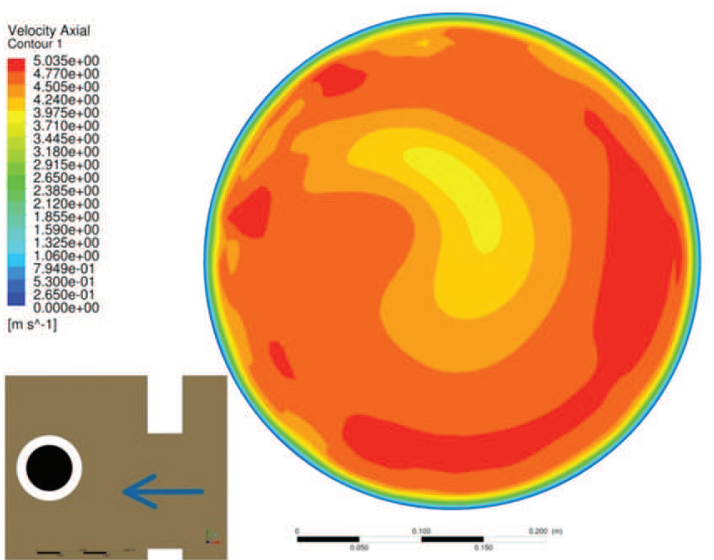

ANSYS

$\stackrel{\leftrightarrow}{\circ}$

Figure 9. Axial velocity on the inlet to the suction cone.

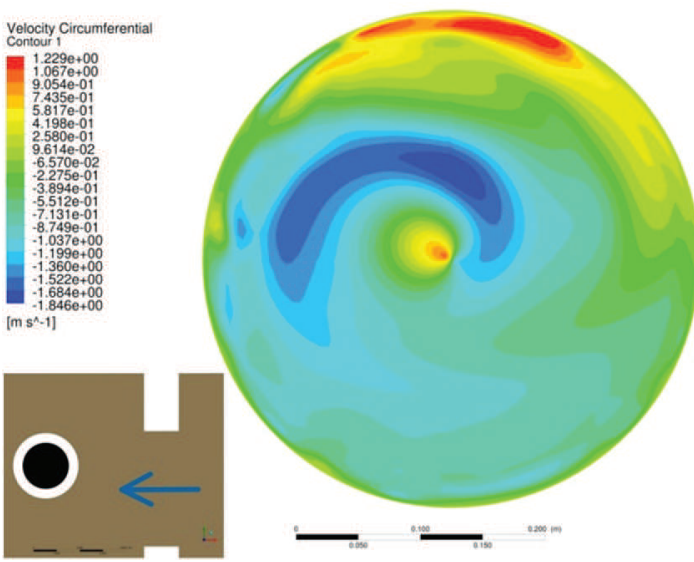

Figure 10. Circumferential velocity on the inlet to the suction cone.

Figure 8. Streamlines visualizing vertical structures for

$\mathrm{Q}=115 \%$ of $\mathrm{BEP}$ flow rate. 

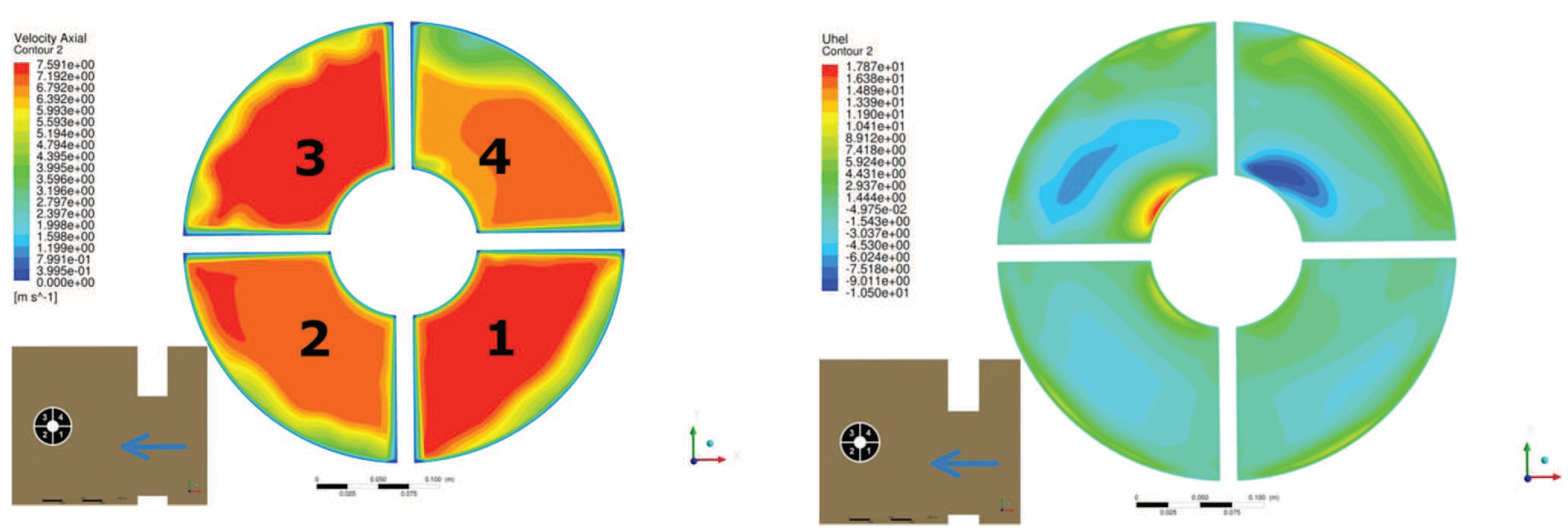

Figure 11 Axial velocity just in front of the pump impeller.

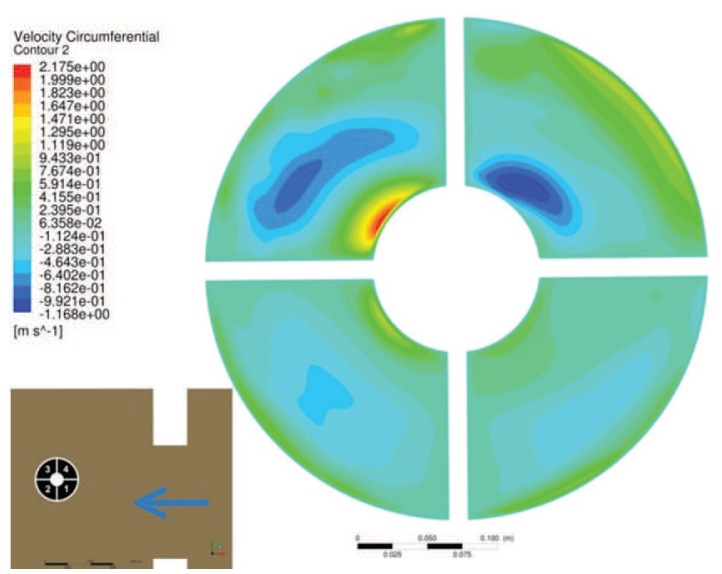

Figure 12. Circumferential velocity just in front of the pump impeller.

Nonuniformity of velocity fields increases with increasing flow rate. Quite strong vortices extend to quadrants 3 and 4 , which are close to the side walls.

From the point of hydraulic machine design it is well known that inlet velocity angles play important role in proper behaviour of the pump impeller. Therefore inlet velocity angles were evaluated and plotted, see figures 13, 14. Again only situation for BEP flow rate is illustrated.

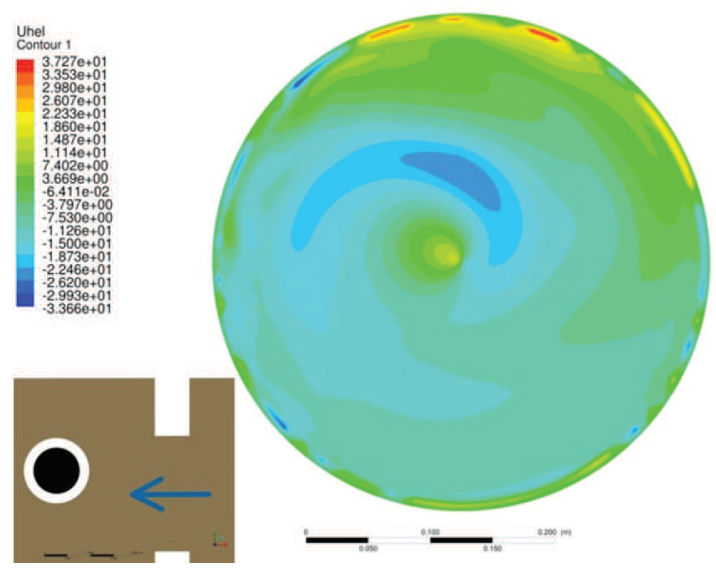

Figure 13. Velocity angle (from the axial direction) on the inlet to the suction cone.
Figure 14. Velocity angle (from the axial direction) just in front of the pump impeller.

Maximum flow angle deviations from purely axial direction at the inlet to suction cone are $+37^{\circ}$ and $33^{\circ}$ respectively. Position of the maximum angle is close to cone walls, but also in vicinity of the axis. Supporting cross has clearly positive effect, because flow deviation angle drops to maximum values of $+17^{\circ}$ and $-10^{\circ}$.

It should be noted that while the numerical simulation was unsteady there were no unsteady effects observed like jumping of the vortices among quadrants of the inlet cross-section or axial flow pulsations.

It was mentioned that three vortices of two types arise in the pump sump. Vortex 1 (surface vortex) shifts to sump corner with increasing flow rate, while vortex 3 (bottom vortex) is pushed away from the pump axis.

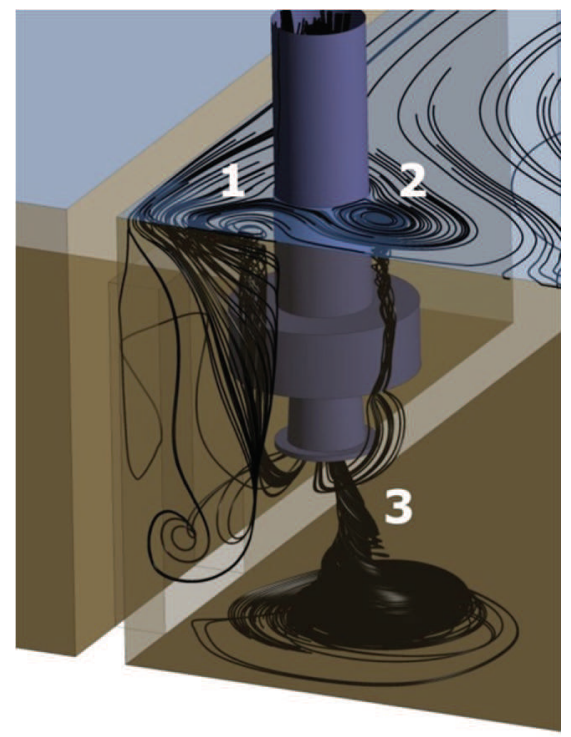

Figure 15. Detail of the pump-inatke vortices.

Distribution of the vortices over the inlet cross-section just in front of the pump impeller for different operating points is depicted in figure 16 . Vector plots confirm that most of the flow non-uniformity occurs in quadrants 3 and 4 and the vortex strength grows with increasing flow rate. 


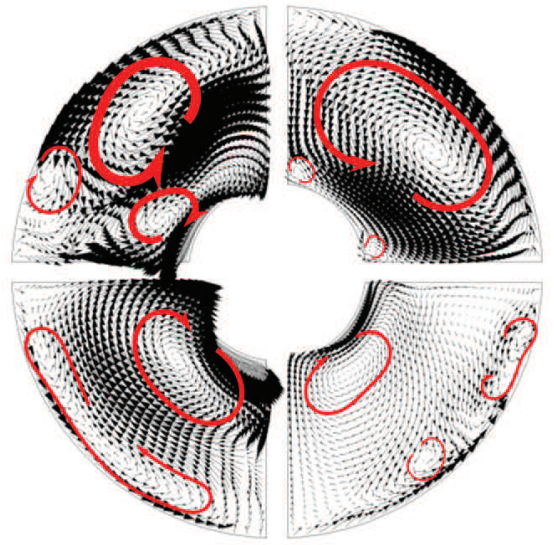

Figure 16. Visualization of the vortical structures just in front of the impeller for $\mathrm{Q}=55 \%$ of BEP flow rate.

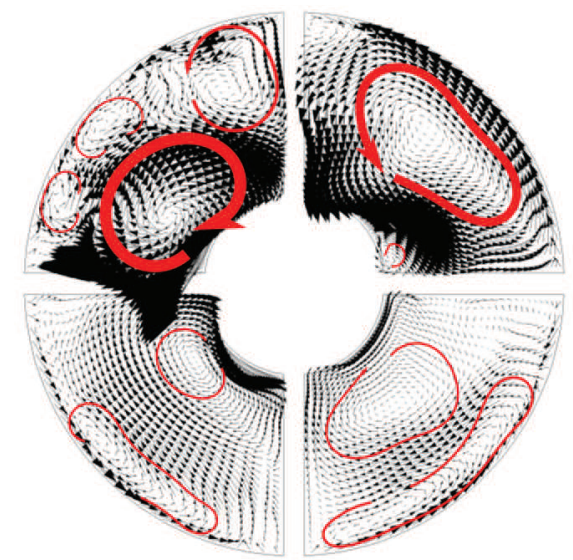

Figure 17. Visualization of the vortical structures just in front of the impeller for $\mathrm{Q}=100 \%$ of BEP flow rate.

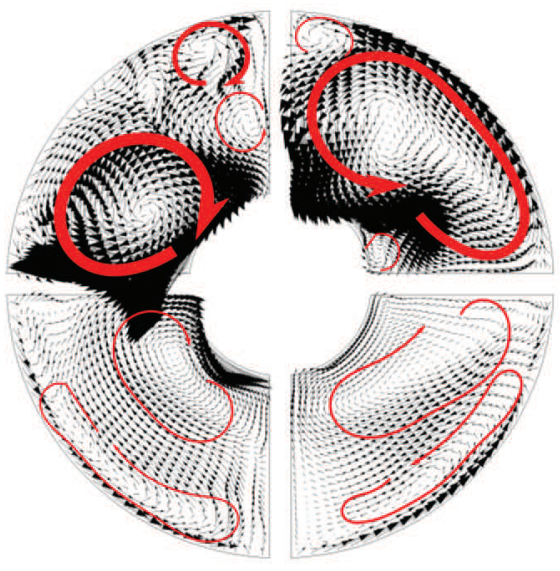

Figure 18. Visualization of the vortical structures just in front of the impeller for $\mathrm{Q}=115 \%$ of BEP flow rate.

\section{Empirical analysis and remedies}

Literature cites several formulas for empirical estimation of the minimum pump submergence to prevent surface vortices. All of them are based on experimental data and submergence depth is function of Froude number.

$$
F \mathrm{r}=\mathrm{v} / \sqrt{\mathrm{gD}},
$$

where $v$ is inlet velocity and $D$ is diameter of the suction cone inlet. Two most frequently used formulas are considered for flow rate corresponding to the best efficiency point.

Knausse [18]:

$$
h=D(2 \cdot F r+0.5)=1.98 m,
$$

Gordon [19]:

$$
h=2.3 \cdot D \cdot F r=2.05 \mathrm{~m} .
$$

Actual submergence of the pump cone inlet is only 1.47 $\mathrm{m}$, which is below both empirical criteria. These results qualitatively support CFD simulations and confirm that appearance of the intake vortices for given boundary conditions is quite likely.

Possible remedies and suggestions to pump operator to mitigate the vortices include:

- $\quad$ floating sieves to suppress the surface vortices 1 and 2

- $\quad$ vertical plate/plates along the side wall to break the surface vortices 1 and 2

- cone with baffles placed on the sump bottom just below the pump suction cone to mitigate bottom vortex $3[4,9]$.

\section{Conclusion}

Computational simulation identified three vortices (two surface vortices, one bottom vortex), which arise due to very asymetric position of the pump within sump, because of proximity of the side walls and bottom and because of the low submergence of the pump suction cross-section.

While vortices do not cause entrainment of air, distortion of the flow and high flow angle deviations may induce shock on impeller blades leading edges and consequently result in blockage of the blade channels or cavitation. US standard ANSI [20] allows maximum level of preswirl to be in interval from $-5^{\circ}$ to $+5^{\circ}$ degrees. Actual installation evokes more than twice higher flow deviation angles.

Highly distorted flow on the inlet may also be responsible for rather complex three-dimensional flow pattern within blade channels and can be propagated even downstream to the guide vane channels and cause the partial flow blockage here.

Although several design modifications and add-on devices, which could eliminate influence of the intake vortices on pump operation were suggested, it should be stressed that proper location of the pump in sump is the primary task of pump station project process. In case that pump is replaced for a new one with increased flow rate while sump dimensions are retained, empirical relations $[2,3]$ have to be checked. If occurence of pump intake vortices is indicated then CFD modeling has to be incorporated to identify position and number of the vortices and to design efficient mitigation strategy. 


\section{Acknowledgement}

This work is an output of research and scientific activities of NETME Centre, regional R\&D centre built with the financial support from the Operational Programme Research and Development for Innovations within the project NETME Centre (New Technologies for Mechanical Engineering), Reg. No. CZ.1.05/2.1.00/01.0002 and, in the follow-up sustainability stage, supported through NETME CENTRE PLUS (LO1202) by financial means from the Ministry of Education, Youth and Sports under the „National Sustainability Programme I“.

Research was also supported by project FSI-S-14-2480 (Innovative fluid machines) of Brno University of Technology, Faculty of Mechanical Engineering.

\section{References}

1. G.M. Jones, R.L. Sanks, G. Tchobanoglous, B.E. Bosserman, Pumping station design (2008)

2. V.P. Rajendran, V.C. Patel, Proc. FEDSM 98 (1998)

3. K. Mansa, Y. Wu, Y. Li, Y. Li, Y. Xu, J. Hydrol. Hydromech. 51, 2, pp. 138-143 (2003)

4. T.C. Sassaman, A.E. Johansson, M. Padmanabhan, ASME 2009 Power conference (2009)

5. G. Möller, PhD thesis, ETH Zürich (2003)

6. D.I. Bauer, T. Nakato, IIHR Tech. rep. 389 (1997)

7. T. Nakato, D.D. Jong, IIHR Tech. rep. 402 (1999)

8. V. Naderi, D. Farsadizadeh, A. H. Dalir, H. Arvanaghi, J. Civil Eng. Urbanism, 3, 6, pp. 368-371 (2013)

9. Z. Ahmad, B. Jain, S. Kumar, M.K. Mittal, J. Pipeline Sys. Eng. Practice (2011)

10. D. W. Free, B.P. Tullis, J.R. Bird, Proc. $16^{\text {th }}$ Int. Pump Users Symp. (1999)

11. A. Skerlavaj, L. Skerget, J. Ravnik, A. Lipej, Proc. Inst. Mech. Eng., Part A: Journal of Power and Energy, 225, 6, 764-778 (2011)

12. T.Y. Tokyay, S. G. Constantinescu, IIHR Tech. rep 445 (2005)

13. F. A Muggli, S. Krüger, Sulzer Tech. Review, 3 (2011)

14. T. Okamura, K. Kamemoto, 8th Asian Int. Fluid Mach. Conf. (2005)

15. A. Issa, A.C. Bayeul, G. Bois, 24th IAHR Symp. (2008)

16. S.G. Constantinescu, V.C. Patel, IIHR Tech. Rep. 394 (1998)

17. P. Rudolf, M. Hudec, P. Zubík, D. Štefan, EPJ Web of Conf., 25 (2012)

18. J. Knauss, Swirling flow problems at intakes, IAHR Hydraulic Structures Design Manual (1987)

19. J.L. Gordon, Water Power, 22, 4, pp. 137-138 (1970)

20. Hydraulic Institute Standards, ANSI/HI 9.8.5.6 (1998) 